PROCEEDINGS OF THE

AMERICAN MATHEMATICAL SOCIETY

Volume 131, Number 8, Pages 2409-2414

S 0002-9939(03)06874-6

Article electronically published on January 15, 2003

\title{
ON A CLASS OF SUBLINEAR QUASILINEAR ELLIPTIC PROBLEMS
}

\author{
D. D. HAI
}

(Communicated by David S. Tartakoff)

\begin{abstract}
We establish existence and multiplicity of positive solutions to the quasilinear boundary value problem

$$
\begin{aligned}
\operatorname{div}\left(|\nabla u|^{p-2} \nabla u\right) & =-\lambda f(u) \text { in } \Omega, \\
u & =0 \quad \text { on } \partial \Omega,
\end{aligned}
$$

where $\Omega$ is a bounded domain in $R^{n}$ with smooth boundary $\partial \Omega, f:[0, \infty) \rightarrow R$ is continuous and p-sublinear at $\infty$, and $\lambda$ is a large parameter.
\end{abstract}

\section{INTRODUCTION}

Consider the quasilinear elliptic boundary value problem

$$
\begin{aligned}
\Delta_{p} u & =-\lambda f(u) \text { in } \Omega, \\
u & =0 \text { on } \quad \partial \Omega,
\end{aligned}
$$

where $\Delta_{p} u=\operatorname{div}\left(|\nabla u|^{p-2} \nabla u\right), p>1, \Omega$ is a bounded domain in $R^{n}$ with smooth boundary $\partial \Omega, f:[0, \infty) \rightarrow R$, and $\lambda$ is a positive parameter.

Problem (I) has been studied extensively during recent years (see [2]-11] and the references therein). In [5], Guo and Webb proved existence results for (I) for $\lambda$ large when $f$ is a smooth, nondecreasing positive function on $(0, \infty), \lim _{s \rightarrow \infty} s^{-\mu} f(s)=$ $\beta>0$ for some $\mu \in(0, p-1)$, and $\liminf _{x \rightarrow 0^{+}} \frac{f(s)}{s^{p-1}}>0$, using Serrin's sweeping principle. They also considered uniqueness of positive solutions when $\frac{f(x)}{x^{p-1}}$ is decreasing on $(0, \infty)$. Multiplicity results were established in [4] when the above condition of $f$ at 0 is replaced by $\lim _{s \rightarrow 0} \frac{f(s)}{s^{p-1}}=0$. Related results for the radial case in a ball using ordinary differential equations techniques can be found in [6]. In this paper, we shall establish existence results to (I) for $\lambda$ large when $f$ is merely continuous and p-sublinear at $\infty$. In particular, we do not assume any conditions of $f$ near 0 , nor monotonicity and smoothness on $f$ as in [4, 5]. We also establish a multiplicity result for (I) under additional assumptions. Our approach depends on $C^{1, \beta}$ regularity results in [7, 9] to create ordered sub- and supersolutions that provide a solution in between via the Schauder fixed point theorem. Note that our sub- and supersolutions are defined in the operator context and are not weak suband supersolutions in general.

Received by the editors March 7, 2002.

2000 Mathematics Subject Classification. Primary 35J25, 35J70.

Key words and phrases. Sub-supersolutions, quasilinear elliptic, positive solutions. 


\section{Existence ReSUlts}

We shall make the following assumptions:

(A.1) $f:[0, \infty) \rightarrow R$ is continuous and there exist positive numbers $K, L_{1}$ such that $f(x)>2 L_{1}$ for $x>K$.

(A.2) $\lim _{x \rightarrow \infty} \frac{f(x)}{x^{p-1}}=0$.

Our main result is

Theorem 1. Let (A.1)-(A.2) hold. Then there exists $\lambda_{0}>0$ such that (I) has a positive solution for $\lambda>\lambda_{0}$. If, in addition, $f \geq 0$ on $(0, \infty)$ and

$$
\lim _{x \rightarrow 0^{+}} \frac{f(x)}{x^{p-1}}=0
$$

then (I) has at least two positive solutions for $\lambda>\lambda_{0}$.

We first need some preliminary results. We shall denote by $\|\cdot\|_{p}$ and $|\cdot|_{p}$ the norms in $L^{p}(\Omega)$ and $C^{p}(\bar{\Omega})$ respectively.

The following lemma gives positivity of the p-Laplacian operator.

Lemma 1 ([10]). Let $x=\left(x_{1}, \ldots, x_{n}\right), y=\left(y_{1}, \ldots, y_{n}\right)$ be two points in $R^{n}$, and let |.| and (.,.) denote the Euclidean norm and the corresponding inner product in $R^{n}$ respectively.

Then

(i) If $p \geq 2$,

$$
\left(|x|^{p-2} x-|y|^{p-2} y, x-y\right) \geq\left(\frac{1}{2}\right)^{p-1}|x-y|^{p} .
$$

(ii) If $1<p \leq 2$,

$$
(|x|+|y|)^{2-p}\left(|x|^{p-2} x-|y|^{p-2} y, x-y\right) \geq(p-1)|x-y|^{2} .
$$

Next, let $\phi \in C^{1}(\bar{\Omega})$ be the solution of

$$
\left\{\begin{array}{c}
\Delta_{p} \phi=-1 \text { in } \Omega \\
\phi=0 \text { on } \partial \Omega .
\end{array}\right.
$$

Then we have

Lemma 2. Let $g \in L^{\infty}(\Omega)$ and let $u \in W_{0}^{1, p}(\Omega)$ satisfy

$$
\left\{\begin{array}{c}
\Delta_{p} u=-g \text { in } \Omega, \\
u=0 \text { on } \partial \Omega .
\end{array}\right.
$$

Then $u \in L^{\infty}(\Omega)$ and

$$
\|u\|_{\infty} \leq M|\phi|_{0}
$$

where $M=\|g\|_{\infty}^{\frac{1}{p-1}}$.

Proof. Since $\Delta_{p}(M \phi)=-M^{p-1}=-\|g\|_{\infty}$ and $\Delta_{p} u=-g$, it follows from the weak maximum principle [8] that $-M \phi \leq u \leq M \phi$, from which the lemma follows.

Lemma 3. Let $C, L_{0}, L_{1}$, and $L$ be positive numbers with $L_{0} \leq L \leq L_{1}$. Suppose that there exist $h, h_{\lambda, L} \in L^{\infty}(\Omega)$ such that

$$
\left\|h_{\lambda, L}\right\|_{\infty} \leq C
$$

for every $\lambda>0, L_{0} \leq L \leq L_{1}$, and

$$
\lim _{\lambda \rightarrow \infty}\left\|h_{\lambda, L}-h\right\|_{2}=0
$$


uniformly for $L \in\left[L_{0}, L_{1}\right]$. Let $u_{\lambda, L}$ and $u$ be solutions of

$$
\Delta_{p} u_{\lambda, L}=-h_{\lambda, L} \text { in } \Omega, u_{\lambda, L}=0 \text { on } \partial \Omega
$$

and

respectively. Then

$$
\Delta_{p} u=-h \text { in } \Omega, u=0 \text { on } \partial \Omega
$$

$$
\lim _{\lambda \rightarrow \infty}\left|u_{\lambda, L}-u\right|_{1}=0
$$

uniformly for $L \in\left[L_{0}, L_{1}\right]$.

Proof. By (2.1) and Lemma 2, it follows that $u_{\lambda, L}$ are bounded in $L^{\infty}(\Omega)$ independent of $\lambda, L$. It then follows from regularity results in [7, 9] that there exist numbers $\beta \in(0,1)$ and $C_{1}>0$ such that $u_{\lambda, L}, u \in C^{1, \beta}(\bar{\Omega})$ and

$$
\left|u_{\lambda, L}\right|_{1}<C_{1}
$$

for all $\lambda>0, L \in\left[L_{0}, L_{1}\right]$.

Multiplying the equation

$$
\Delta_{p} u_{\lambda, L}-\Delta_{p} u=-\left(h_{\lambda, L}-h\right)
$$

by $u_{\lambda, L}-u$ and integrating gives

$$
\begin{aligned}
& \int_{\Omega}\left(\left|\nabla u_{\lambda, L}\right|^{p-2} \nabla u_{\lambda, L}-|\nabla u|^{p-2} \nabla u, \nabla u_{\lambda, L}-\nabla u\right) d x \\
& \quad=\int_{\Omega}\left(h_{\lambda, L}-h\right)\left(u_{\lambda, L}-u\right) d x \\
& \quad \leq\left\|h_{\lambda, L}-h\right\|_{2}\left\|u_{\lambda, L}-u\right\|_{2} .
\end{aligned}
$$

On the other hand, Lemma 1 and (2.3) imply

$$
\int_{\Omega}\left(\left|\nabla u_{\lambda, L}\right|^{p-2} \nabla u_{\lambda, L}-|\nabla u|^{p-2} \nabla u, \nabla u_{\lambda, L}-\nabla u\right) d x \geq C_{2}|| \nabla\left(u_{\lambda, L}-u\right) \|_{2}^{q},
$$

where $q=\max (p, 2)$, and $C_{2}$ is a positive constant depending only on $p, C_{1}$.

Combining (2.4), (2.5), and Poincare's inequality, we obtain

$$
\lim _{\lambda \rightarrow \infty}\left\|u_{\lambda, L}-u\right\|_{2}=0
$$

uniformly for $L \in\left[L_{0}, L_{1}\right]$.

Suppose to the contrary that (2.2) does not hold. Then there exist a positive number $\varepsilon$ and sequences $\left(L_{n}\right) \subset\left[L_{0}, L_{1}\right],\left(\lambda_{n}\right) \rightarrow \infty$ such that

$$
\left|u_{\lambda_{n}, L_{n}}-u\right|_{1} \geq \varepsilon
$$

for all $n$. Since $\left(u_{\lambda_{n}, L_{n}}\right)$ is bounded in $C^{1, \beta}(\bar{\Omega})$, there exist $v \in C^{1}(\bar{\Omega})$ and a subsequence of $\left(u_{\lambda_{n}, L_{n}}\right)$, which we still denote by $\left(u_{\lambda_{n}, L_{n}}\right)$, such that

$$
\lim _{n \rightarrow \infty}\left|u_{\lambda_{n}, L_{n}}-v\right|_{1}=0 .
$$

From this and (2.6), we deduce that $u=v$, a contradiction. This completes the proof of Lemma 3.

For each $\lambda>0$, define the operator $A_{\lambda}$ on $C(\bar{\Omega})$ by $A_{\lambda} v=u$ if

$$
\Delta_{p} u=-\lambda f(v) \text { in } \Omega, u=0 \text { on } \partial \Omega,
$$

where we define $f(x)=f(0)$ if $x<0$. Then $A_{\lambda}: C(\bar{\Omega}) \rightarrow C(\bar{\Omega})$ is completely continuous, and fixed points of $A_{\lambda}$ are solutions of (I). 
The next lemma produces subsolutions to (I).

Lemma 4. Let $0<L_{0} \leq L \leq L_{1}$, where $L_{1}$ is given by (A.1), and let $\phi_{\lambda, L}=$ $(\lambda L)^{\frac{1}{p-1}} \phi$. Then there exists a positive number $\lambda_{0}$ independent of $L$ such that for $\lambda \geq \lambda_{0}$

$$
v \geq \phi_{\lambda, L} \Rightarrow A_{\lambda} v \geq \phi_{\lambda, L}
$$

Proof. Let $v \in C(\bar{\Omega})$ with $v \geq \phi_{\lambda, L}$, and let $u_{\lambda}=A_{\lambda} v, \tilde{u}_{\lambda, L}=\frac{u_{\lambda}}{(2 \lambda L)^{\frac{1}{p-1}}}$. Then

$$
v(x) \geq\left(\lambda L_{0}\right)^{\frac{1}{p-1}} \phi(x)>K \text { if } \phi(x)>\frac{K}{\left(\lambda L_{0}\right)^{\frac{1}{p-1}}},
$$

where $K$ is given by (A.1). Define

$$
h_{\lambda, L}(x)=\left\{\begin{array}{l}
1 \quad \text { if } \phi(x)>\frac{K}{\left(\lambda L_{0}\right)^{\frac{1}{p-1}}}, \\
\frac{M_{0}}{2 L} \text { if } \phi(x) \leq \frac{K}{\left(\lambda L_{0}\right)^{\frac{1}{p-1}}},
\end{array}\right.
$$

where $M_{0}$ is a number such that $f(x) \geq M_{0}$ for all $x$.

By (A.1),

$$
\Delta_{p}\left(\tilde{u}_{\lambda, L}\right)=-\frac{f(v)}{2 L}<-h_{\lambda, L} \text { in } \Omega,
$$

and the maximum principle implies that $\tilde{u}_{\lambda, L} \geq \bar{u}_{\lambda, L}$, where $\bar{u}_{\lambda, L}$ is the solution of

$$
\Delta_{p}\left(\bar{u}_{\lambda, L}\right)=-h_{\lambda, L} \text { in } \Omega, \bar{u}_{\lambda, L}=0 \text { on } \partial \Omega .
$$

Note that

$$
\left\|h_{\lambda, L}-1\right\|_{2} \leq\left(\frac{\left|M_{0}\right|}{2 L}+1\right)\left(\iint_{x \in \Omega: \phi(x) \leq \frac{K}{\left(\lambda L_{0}\right)^{\frac{1}{p-1}}}} d x\right)^{\frac{1}{2}} \rightarrow 0
$$

as $\lambda \rightarrow \infty$ uniformly for $L \in\left[L_{0}, L_{1}\right]$. Since $\Delta_{p} \phi=-1$ in $\Omega$, it follows from Lemma 3 that

$$
\lim _{\lambda \rightarrow \infty}\left|\bar{u}_{\lambda, L}-\phi\right|_{1}=0
$$

uniformly for $L \in\left[L_{0}, L_{1}\right]$. Let $\gamma>0$. Then by the mean value theorem

$$
\left|\bar{u}_{\lambda, L}(x)-\phi(x)\right| \leq\left|\bar{u}_{\lambda, L}-\phi\right|_{1} d(x, \partial \Omega)<\gamma c \phi(x), x \in \Omega,
$$

for $\lambda$ large, where $c>0$ is such that $d(x, \partial \Omega) \leq c \phi(x)$ for all $x \in \Omega$. Hence for such $\lambda$,

$$
\bar{u}_{\lambda, L}(x) \geq(1-\gamma c) \phi(x), x \in \Omega,
$$

from which it follows that

$$
u_{\lambda}(x) \geq(2 \lambda L)^{\frac{1}{p-1}} \bar{u}_{\lambda, L}(x) \geq(\lambda L)^{\frac{1}{p-1}} \phi(x)=\phi_{\lambda, L}(x), x \in \Omega,
$$

if $\gamma$ is sufficiently small. This completes the proof of Lemma 4 .

We are now in a position to give the

Proof of Theorem 1. Let $\lambda>\lambda_{0}$, where $\lambda_{0}$ is given by Lemma 4 .

Let $\bar{\phi}_{\lambda}=M_{\lambda} \phi$ where $M_{\lambda}>>1$. Let $v \in C(\bar{\Omega})$ satisfy $v \leq \bar{\phi}_{\lambda}$. We claim that $\tilde{u}_{\lambda} \equiv A_{\lambda} v \leq \bar{\phi}_{\lambda}$. Indeed, let $\tilde{f}(x)=\sup _{z \leq x} f(z)$. Then $\tilde{f}$ is nondecreasing and

$$
\Delta_{p} \tilde{u}_{\lambda}=-\lambda f(v) \geq-\lambda \tilde{f}\left(M_{\lambda}|\phi|_{0}\right) \geq-M_{\lambda}^{p-1} \text { in } \Omega,
$$


where we use the fact that $\lim _{x \rightarrow \infty} \frac{\tilde{f}(x)}{x^{p-1}}=0$. Since $\Delta_{p}\left(\bar{\phi}_{\lambda}\right)=-M_{\lambda}^{p-1}$, the maximum principle implies $\tilde{u}_{\lambda} \leq \bar{\phi}_{\lambda}$, as claimed.

Thus $A_{\lambda}:\left[\phi_{\lambda, L}, \bar{\phi}_{\lambda}\right] \rightarrow\left[\phi_{\lambda, L}, \bar{\phi}_{\lambda}\right]$, where $\phi_{\lambda, L}$ is defined in Lemma 4 . The Schauder fixed point theorem then gives a fixed point $u$ of $A_{\lambda}$ in $\left[\phi_{\lambda, L}, \bar{\phi}_{\lambda}\right]$, which is a positive solution of (I). This completes the proof of the first part of Theorem 1. Suppose next that $f \geq 0$ and $\lim _{x \rightarrow 0^{+}} \frac{f(x)}{x^{p-1}}=0$. Let $\phi_{\varepsilon}=\varepsilon \phi$. We claim that for $\varepsilon>0$ small,

Indeed, we have

$$
v \leq \phi_{\varepsilon} \Rightarrow u_{\lambda} \equiv A_{\lambda} v \leq \phi_{\varepsilon}
$$

$$
\Delta_{p} u_{\lambda}=-\lambda f(v) \text { in } \Omega
$$

and for $\varepsilon>0$ small,

$$
\lambda f(v) \leq \lambda \tilde{f}(v) \leq \lambda \tilde{f}\left(\varepsilon|\phi|_{0}\right) \leq \varepsilon^{p-1}=-\Delta_{p} \phi_{\varepsilon} \text { in } \Omega,
$$

where $\tilde{f}(x)=\sup _{z \leq x} f(z)$. Here we have used the fact that $\lim _{x \rightarrow \infty} \frac{\tilde{f}(x)}{x^{p-1}}=0$. By the maximum principle, $u_{\lambda} \leq \phi_{\varepsilon}$, as claimed. By decreasing $\varepsilon$ if necessary, we assume $\phi_{\varepsilon}<\phi_{\lambda, L_{0}}$ in $\Omega$. Define

$$
X=C_{0}^{1}(\bar{\Omega}), X_{1}=\left[0, \phi_{\varepsilon}\right], X_{2}=\left[\phi_{\lambda, L_{0}}, \bar{\phi}_{\lambda}\right], \text { and } X_{3}=\left[0, \bar{\phi}_{\lambda}\right],
$$

where $[z, w]=\{u \in X: z \leq u \leq w\}$. Then $A_{\lambda}: X_{k} \rightarrow X_{k}$ and $A_{\lambda}$ has a fixed point $\tilde{u}_{2}$ in $X_{2}$. By the maximum principle [11, every nontrivial fixed point of $A_{\lambda}$ in $X_{3}$ is a positive solution of $(\mathrm{I})$. Since 0 is an interior point of $X_{1}$ with respect to $X_{3}$, if $A_{\lambda}$ has a fixed point $u_{1}$ on the boundary of $X_{1}$ in $X_{3}$, then $0<u_{1}<\tilde{u}_{2}$ in $\Omega$ and thus (I) has two distinct positive solutions. Since $A_{\lambda}:\left[\phi_{\lambda, L_{1}}, \bar{\phi}_{\lambda}\right] \rightarrow\left[\phi_{\lambda, L_{1}}, \bar{\phi}_{\lambda}\right], A_{\lambda}$ has a fixed point $\tilde{u}$ in $\left[\phi_{\lambda, L_{1}}, \bar{\phi}_{\lambda}\right]$. Note that every point in $\left[\phi_{\lambda, L_{1}}, \bar{\phi}_{\lambda}\right]$ is an interior point of $X_{2}$ in $X_{3}$. If $A_{\lambda}$ has a fixed point $u_{2}$ on the boundary of $X_{2}$ in $X_{3}$, then $u_{2} \neq \tilde{u}$ and we have two solutions. Finally, if $A_{\lambda}$ does not have any fixed points on the boundaries of $X_{1}$ and $X_{2}$ in $X_{3}$, then by a result of Amann [1, there exists a fixed point $\bar{u}$ of $A_{\lambda}$ such that $\bar{u} \in X_{3} \backslash\left(X_{1} \cup X_{2}\right)$. In particular, $\bar{u}$ is a second positive solution of (I). This completes the proof of Theorem 1.

Remark 1. (i) Conditions $\lim _{x \rightarrow \infty} \frac{f(x)}{x^{p-1}}=0$ and $\lim _{x \rightarrow 0^{+}} \frac{f(x)}{x^{p-1}}=0$ in Theorem 1 can be replaced by $\liminf \operatorname{in}_{x \rightarrow \infty} \frac{f(x)}{x^{p-1}}=0$ and $\liminf _{x \rightarrow 0^{+}} \frac{f(x)}{x^{p-1}}=0$ respectively, provided that $f$ is nondecreasing.

(ii) The multiplicity result in Theorem 1 was established in [4] under additional assumptions that $f \in C^{1}\left(R^{+}\right)$is strictly increasing on $R^{+}$, and there exist $\alpha_{1}, \alpha_{2}>$ 0 such that $f(s) \leq \alpha_{1}+\alpha_{2} s^{\mu}$ for some $\mu \in(0, p-1)$.

\section{REFERENCES}

[1] H. Amann, Fixed point equations and nonlinear eigenvalue problems in ordered Banach spaces, Siam Rev. 18 (1976), 620-709. MR 54:3519] errata MR 57:7269

[2] P. Drabek and J. Hernandez, Existence and uniqueness of positive solutions for some quasilinear elliptic problems, Nonlinear Anal. 44 (2001), 189-204. MR 2001m:35104

[3] P. Drabek, A. Kufner, and F. Nicolosi, Quasilinear Elliptic Equations with Degenerations and Singularities, de Gruyter Series in Nonlinear Analysis and Applications, Vol. 5, Berlin, New York, 1997. MR 98k:35068

[4] Z. M. Guo, Some existence and multiplicity results for a class of quasilinear eigenvalue problems, Nonlinear Anal. 18 (1992), 957-971. MR 93c:35045

[5] Z. M. Guo and J. R. L. Webb, Uniqueness of positive solutions for quasilinear elliptic equations when a parameter is large, Proc. Roy. Soc. Edinburgh 124 A (1994), 189-198. MR 95a:35046 
[6] D. D. Hai and R. Shivaji, Existence and uniqueness for a class of quasilinear elliptic boundary value problems (submitted)

[7] G. M. Lieberman, Boundary regularity for solutions of degenerate elliptic equations, Nonlinear Anal. 12 (1988), 1203-1219. MR 90a:35098

[8] S. Sakaguchi, Concavity properties of solutions to some degenerate quasilinear elliptic Dirichlet problems, Ann. Scula Norm. Sup. Pisa Cl. Sci. 14 (1987), 403-421. MR 89h:35133

[9] P. Tolksdorf, Regularity for a more general class of quasilinear elliptic equations, J. Diff. Eqns. 51 (1984), 126-150. MR 85g:35047

[10] T. Oden, Qualitative Methods in Nonlinear Mechanics, Prentice-Hall, Inc., Englewood Cliffs, New Jersey, 1986.

[11] J. L. Vazquez, A strong maximum principle for some quasilinear elliptic equations, Appl. Math. Optim. 12 (1984), 191-202. MR 86m:35018

Department of Mathematics, Mississippi State University, Mississippi State, MissisSIPPI 39762

E-mail address: dang@ra.msstate.edu 